\title{
Comparison between the AFNOR Method and the Imposition of Balanced Maximum Specific Sliding in the Imposed Centre Distance Problem
}

\author{
Pedro Freitas ${ }^{1}$, António Francisco Tenreiro ${ }^{2}$, Paulo M. S. T. de Castro ${ }^{3}$ \\ ${ }^{1}$ Faculty of Engineering University of Porto, Porto, Portugal (up201304518@fe.up.pt); \\ ${ }^{2}$ Faculty of Engineering University of Porto, Porto, Portugal (up201304383@fe.up.pt); \\ ${ }^{3}$ Faculty of Engineering University of Porto, Porto, Portugal (ptcastro@fe.up.pt) ORCID 0000- \\ 0003-3202-1343
}

\begin{abstract}
Successive editions of Henriot's treatise on gears, and an AFNOR document, present an approximate procedure for the choice of profile shift values for the pinion and wheel when a center distance value is imposed in a cylindrical gear pair. That procedure aims at achieving an approximate balancing of the maximum specific sliding values of the pinion and of the wheel. The method involves a loosely defined choice of an auxiliary parameter, but no information is available relating this choice with the level of attainment of the intended balancing of maximum specific sliding values. This assessment, if needed, requires a subsequent analysis for verification.

Since no information is available evaluating the procedure, the purpose of this work is to provide a thorough rigorous analysis of the method, highlighting its qualities but also its shortcomings.
\end{abstract}

Author Keywords. Gear Geometry, Profile Shift, Specific Sliding

Type: Research Article

๖ Open Access $\square$ Peer Reviewed @i) cC BY

\section{Introduction}

Imposition of a given center distance, $a^{\prime}$, involves the selection of appropriate profile shift values $x_{1}$ (pinion), and $x_{2}$ (wheel). For a given choice of center distance, $a^{\prime}$, this selection may be made in accordance with an approximate, simplified procedure discussed by Henriot and published by AFNOR. This work aims at evaluating this procedure in light of the specific sliding of the pinion and wheel, $g_{s 1}$ and $g_{s 2}$, respectively. The approximate solution mentioned is compared with the analytical solution for balancing the maximum specific sliding values.

In the present paper it is intended to assess the quality of approximation involved in the use of the simplified solution, since such evaluation is not explicitly available in the technical literature.

This article begins with a brief introduction stating the problem and presenting the methodology used to determine the profile shift values, $x_{1}$ and $x_{2}$, in the imposed center distance problem. This is followed by the problem formulation, establishing an alternative approach to determine $x_{1}$ and $x_{2}$.

The outcomes of the two considered approaches are discussed, with an emphasis on values of $x_{1}, x_{2}, g_{s 1}$ and $g_{s 2}$. Finally, the conclusions obtained are presented. 


\section{The problem and its context}

In the meshing of two toothed wheels, there is sliding between the teeth. This can be verified through the equations (1) and (2). For a more detailed study, the reader is directed to the treatise 'Engrenages: conception, fabrication et mise-en-oeuvre' by Georges Henriot.

$$
\begin{aligned}
& g s_{1, \text { max }}=\left|1-\frac{\sqrt{\left(\frac{z_{2} m}{2}+m+x_{2} m\right)^{2}-r b_{2}{ }^{2}}}{\left(r b_{1}+r b_{2}\right) \tan \left(\alpha^{\prime}\right)-\sqrt{\left(\frac{z_{2} m}{2}+m+x_{2} m\right)^{2}-r b_{2}{ }^{2}}} \cdot \frac{z_{1}}{z_{2}}\right| \\
& g s_{2, \text { max }}=\left|\frac{\sqrt{\left(\frac{z_{1} m}{2}+m+x_{1} m\right)^{2}-r b_{1}{ }^{2}}}{\left(r b_{1}+r b_{2}\right) \tan \left(\alpha^{\prime}\right)-\sqrt{\left(\frac{z_{1} m}{2}+m+x_{1} m\right)^{2}-r b_{1}{ }^{2}}} \cdot \frac{z_{2}}{z_{1}}-1\right|
\end{aligned}
$$

According to Henriot, when the sum of the number of teeth of the pinion and of the wheel is equal or higher than 60 , it can be seen that the profile shift value for the pinion, $x_{1}$, is symmetrical to the value for the wheel, $x_{2}$. This implies that there is no variation of center distance.

$$
z_{1}+z_{2} \geq 60 \Leftrightarrow x_{1}=-x_{2} \Leftrightarrow a^{\prime}=a
$$

In case the sum, $z_{1}+z_{2}$, is less than 60 , the relationship (3) is no longer applicable, and $a^{\prime} \neq a$, so

$$
z_{1}+z_{2}<60 \Leftrightarrow x_{1} \neq x_{2} \Leftrightarrow a^{\prime} \neq a
$$

Profile shift values may be obtained from graphs, as found in the mentioned book 'Engrenages: conception, fabrication et mise en-oeuvre' by Georges Henriot. In consequence, the center distance value $a^{\prime}$ may be calculated as

$$
\left\{\begin{array}{c}
a \cos (\alpha)=a^{\prime} \cos \left(\alpha^{\prime}\right) \\
\operatorname{inv}\left(\alpha^{\prime}\right)=\operatorname{inv}(\alpha)+2 \tan (\alpha) \frac{x_{1}+x_{2}}{z_{1}+z_{2}}
\end{array}\right.
$$

The inverse problem, so-called 'imposed centre distance problem', occurs when the value of centre distance is imposed. In this case, it is necessary to determine the new pressure angle, $\alpha^{\prime}$, as well as the profile shift values, $x_{1}$ and $x_{2}$. However, there are three unknowns for two equations, in which the imposed pressure angle, $\alpha^{\prime}$, and the sum of profile shift values, $\Sigma x=$ $x_{1}+x_{2}$, may be obtained. There remains, however, the question of determining separately the profile shifts, $x_{1}$ and $x_{2}$ (Henriot 2007, 2002c, 2002b, 2002a).

To address this problem, for reduction gears, the AFNOR NF-E23-013 document 'Déport des dentures de roues cylindriques pour engrenages réducteurs' presents a third equation, of empirical nature, allowing to determine the values of $x_{1}$ and $x_{2}$.

$$
x_{1}=\lambda \cdot \frac{i-1}{i+1}+\sum x \cdot \frac{1}{i+1}
$$

where $i=z_{2} / z_{1}$ is the transmission ratio and, $\lambda$, is a coefficient that varies between 0.5 and 0.75 , depending on whether it is a gear pair with a high or a low value of $(z 1+z 2)$, respectively. The equation (6) can be rewritten such that

$$
x_{1}=\lambda \cdot \frac{z_{2}-z_{1}}{z_{2}+z_{1}}+\sum x \cdot \frac{z_{1}}{z_{2}+z_{1}}
$$

In this study, the addendum (in French, saillie) is always taken as $m+x \cdot m$.

It should be noted that, according to Henriot, this procedure is called 'Procedure ISO'; nevertheless, it has no direct reference to an ISO standard. The AFNOR document NF-E23013, which presents the simplified procedure, mentions that there is a study being carried out by ISO with a view on publishing a technical report instead of a standard. The Association Française de Normalization decided to publish this technical study in 1980, as a fascicule de 
documentation, in order to make available a tool to solve this type of problem. However, since that date, ISO has not published any technical standard or report on the choice of profile shift for gears with imposed center distance (AFNOR 1980; Henriot 2007, 2002c, 2002a).

\section{Imposed center distance in cylindrical gear pairs}

Since the system of equations in (5) has three unknowns, $x_{1}, x_{2}$ and $\alpha^{\prime}$, and only two equations, a third equation is therefore necessary in order to obtain an analytical solution. $\mathrm{A}$ possible criterion for arriving at an analytical solution is the imposition of balanced maximum specific sliding in the two wheels. As found in (1) and (2), we have

$$
\begin{aligned}
& g s_{1, \text { max }}=\left|1-\frac{\sqrt{\left(\frac{z_{2} m}{2}+m+x_{2} m\right)^{2}-r b_{2}{ }^{2}}}{\left(r b_{1}+r b_{2}\right) \tan \left(\alpha^{\prime}\right)-\sqrt{\left(\frac{z_{2} m}{2}+m+x_{2} m\right)^{2}-r b_{2}{ }^{2}}} \cdot \frac{z_{1}}{z_{2}}\right| \\
& g s_{2, \text { max }}=\left|\frac{\sqrt{\left(\frac{z_{1} m}{2}+m+x_{1} m\right)^{2}-r b_{1}{ }^{2}}}{\left(r b_{1}+r b_{2}\right) \tan \left(\alpha^{\prime}\right)-\sqrt{\left(\frac{z_{1} m}{2}+m+x_{1} m\right)^{2}-r b_{1}{ }^{2}}} \cdot \frac{z_{2}}{z_{1}}-1\right|
\end{aligned}
$$

The expressions for the maximum values of specific sliding may be transformed so that they do not include the module,

$$
\begin{aligned}
& g s_{1, \max }=\left|1-\frac{\sqrt{\left(\frac{z_{2}}{2}+1+x_{2}\right)^{2}-\left(\frac{z_{2} \cos (\alpha)}{2}\right)^{2}}}{\left(z_{1}+z_{2}\right) \frac{\cos (\alpha)}{2} \tan \left(\alpha^{\prime}\right)-\sqrt{\left(\frac{z_{2}}{2}+1+x_{2}\right)^{2}-\left(\frac{z_{2} \cos (\alpha)}{2}\right)^{2}}} \cdot \frac{z_{1}}{z_{2}}\right| \\
& g s_{2, \text { max }}=\left|\frac{\sqrt{\left(\frac{z_{1}}{2}+1+x_{1}\right)^{2}-\left(\frac{z_{1} \cos (\alpha)}{2}\right)^{2}}}{\left(z_{1}+z_{2}\right) \frac{\cos (\alpha)}{2} \tan \left(\alpha^{\prime}\right)-\sqrt{\left(\frac{z_{1}}{2}+1+x_{1}\right)^{2}-\left(\frac{z_{1} \cos (\alpha)}{2}\right)^{2}}} \cdot \frac{z_{2}}{z_{1}}-1\right|
\end{aligned}
$$

Imposing $g s_{1, \max }=g s_{2, \max }$ leads to an equation in $x_{1}$ and $x_{2}$, where $x_{2}$ may be written as $x_{2}=\Sigma x$ - $x_{1}$. After numerical solution of this equation, where the sole unknown is $x_{1}$, the value of $x_{2}$ is obtained from $x_{2}=\sum x-x_{1}$. Obviously, the procedure could be carried out using as unknown $x_{2}$, and finally obtaining $x_{1}$ as $x_{1}=\sum x-x_{2}$.

\section{Results and Discussion}

For a variety of gear pairs and center distance values, the results of $x_{1}, x_{2}$ and $g s_{\max }$ obtained analytically as well as obtained with the suggested approximation are given below.

It should be noted that center distance variation, $\Delta a$, was defined as

$$
\Delta a=\frac{a^{\prime}-a}{a} \times 100[\%]
$$

Figures 1,4 and 7 show the specific sliding values of the pinion, $g s_{1, \max }$, and of the wheel, $g s_{2, \max }$, as a function of the center-distance variation, using the AFNOR procedure.

Figures 2, 5 and 8 show (i) the specific sliding of the two wheels, gsig,max, when using the criterion of balancing maximum values of $g s$ as well as (ii) the maximum specific sliding, $g s_{A F N O R, \max }$ as a function of center distance variation, $\Delta a$. The maximum specific sliding, $g_{\text {SAFNOR, max }}$, is defined as

$$
g s_{A F N O R ; \max }=\max \left\{g s_{1, \max } ; g s_{2, \max }\right\}
$$


Figures 3, 6 and 9 show the profile shift values $x_{1}$ and $x_{2}$, when using the criterion of balancing maximum values of specific sliding, as well as the maximum specific sliding as a function of $\Delta a$.

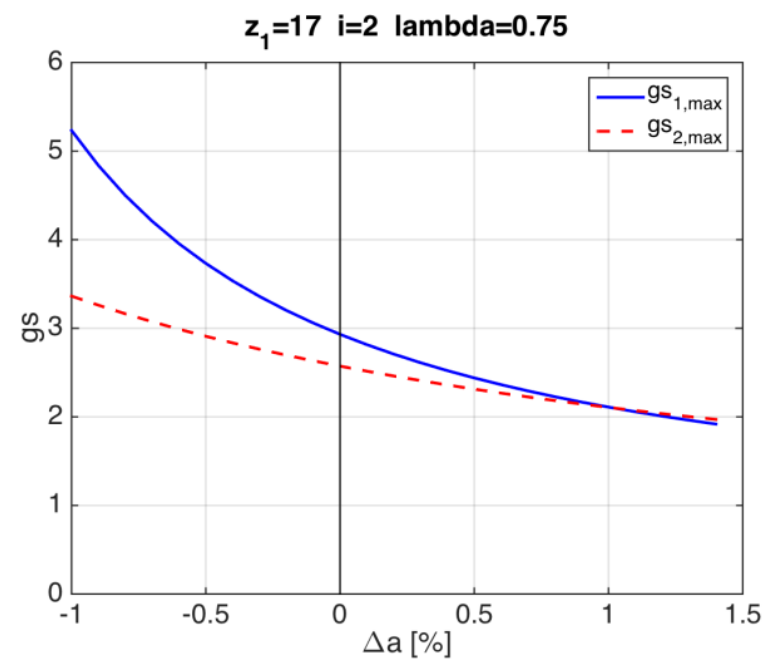

Figure 1: Specific sliding values of pinion and wheel, as a function of the center-distance variation, $\Delta a$, using the AFNOR procedure, for a pinion with 17 teeth and a speed ratio of 2 $z_{1}=17 i=2$ lambda $=0.75$

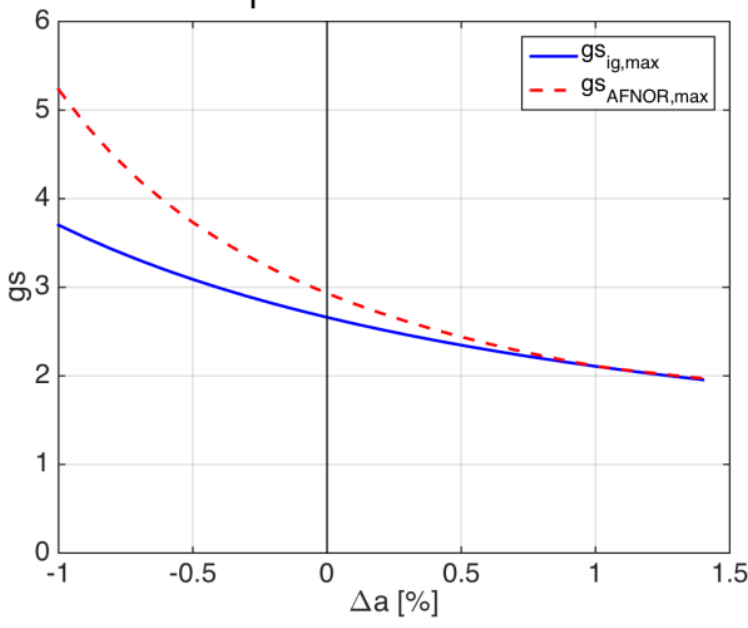

Figure 2: Specific sliding of the two wheels when using the criterion of balancing maximum values of $g s$ as well as the maximum specific sliding as a function of center distance variation, $\Delta a$, for a pinion with 17 teeth and a speed ratio of 2

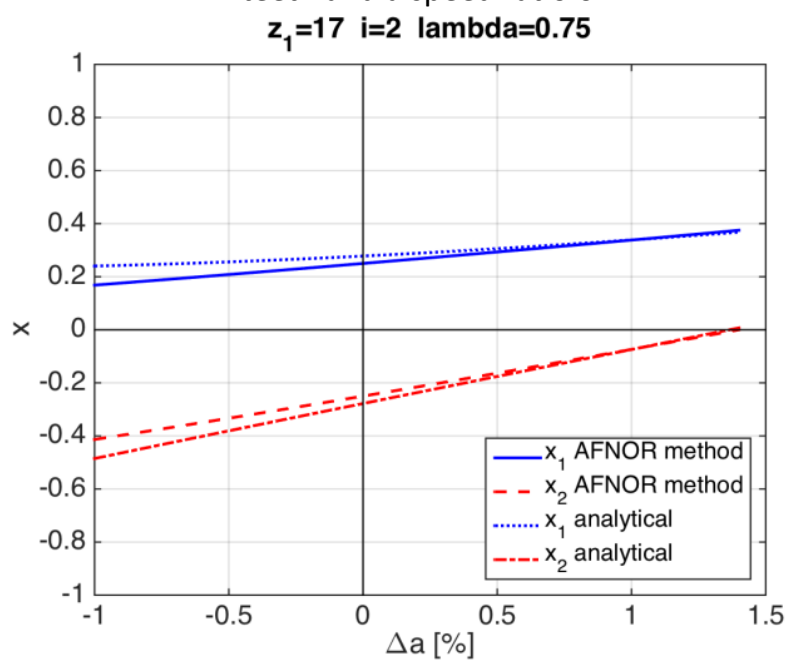

Figure 3: Profile shift values, $x_{1}$ and $x_{2}$, when using the criterion of balancing maximum values of specific sliding, as well as the maximum specific sliding, as a function of $\Delta a$, for a pinion with 17 teeth and a speed ratio of 2 


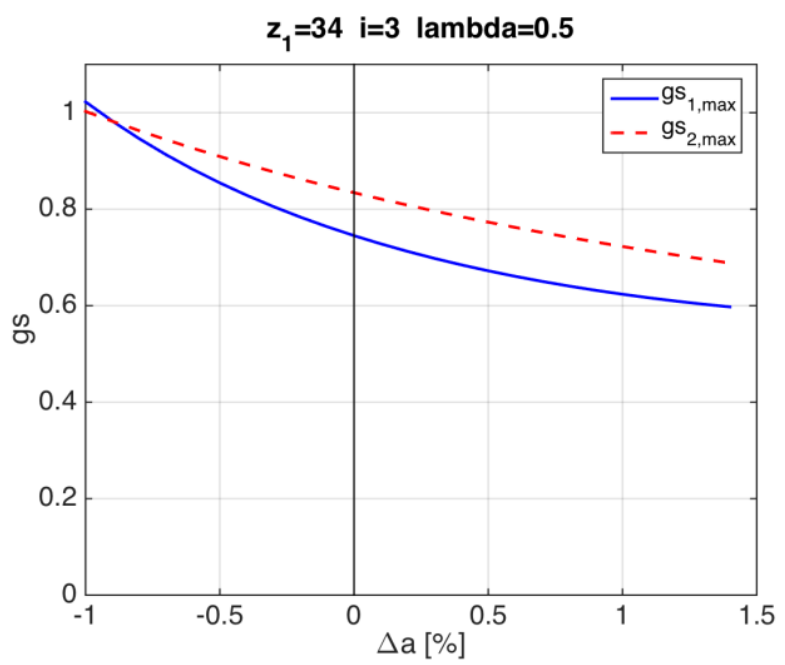

Figure 4: Specific sliding values of pinion and wheel, as a function of the center-distance variation, $\Delta a$, using the AFNOR procedure, for a pinion with 34 teeth and a speed ratio of 3 $z_{1}=34 i=3$ lambda $=0.5$

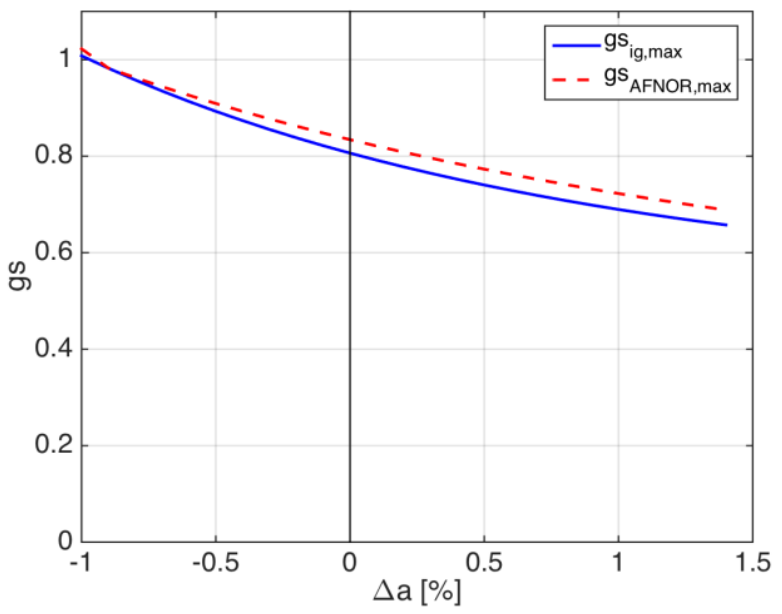

Figure 5: Specific sliding of the two wheels when using the criterion of balancing maximum values of $g s$ as well as the maximum specific sliding as a function of center distance variation, $\Delta a$, for a pinion with 34 teeth and a speed ratio of 3

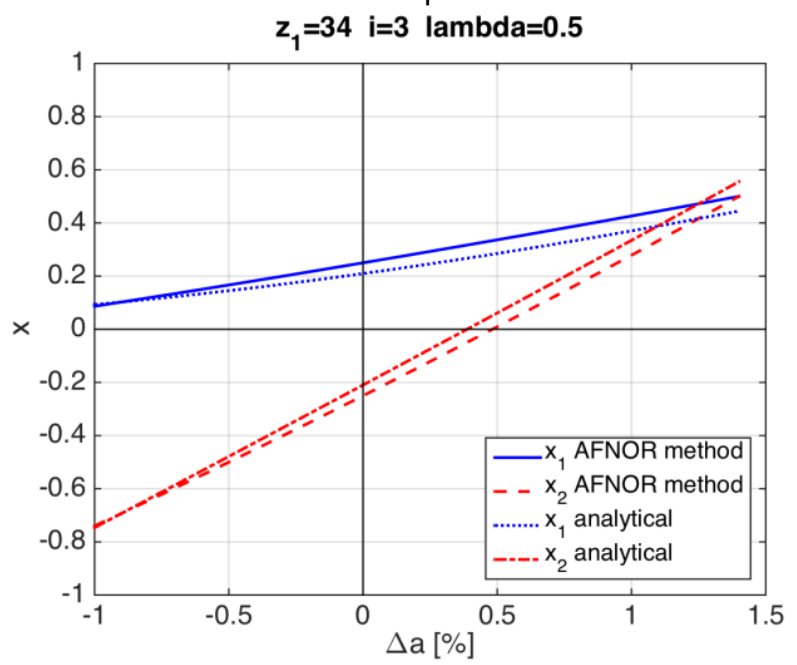

Figure 6: Profile shift values, $x_{1}$ and $x_{2}$, when using the criterion of balancing maximum values of specific sliding, as well as the maximum specific sliding, as a function of $\Delta a$, for a pinion with 34 teeth and a speed ratio of 3 


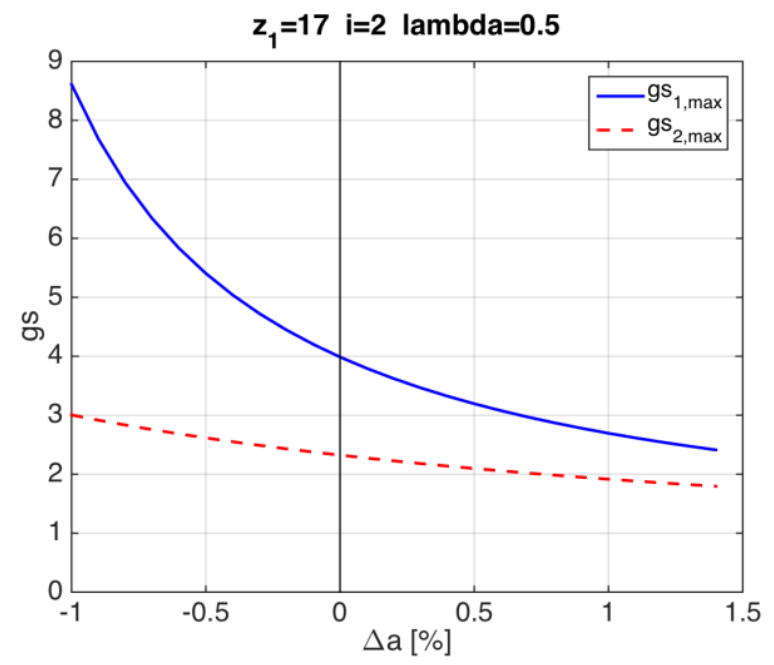

Figure 7: Specific sliding values of pinion and wheel, as a function of the center-distance variation, $\Delta a$, using the AFNOR procedure, for a pinion with 17 teeth and a speed ratio of 2

$\mathrm{z}_{1}=17 \mathrm{i}=2$ lambda $=0.5$

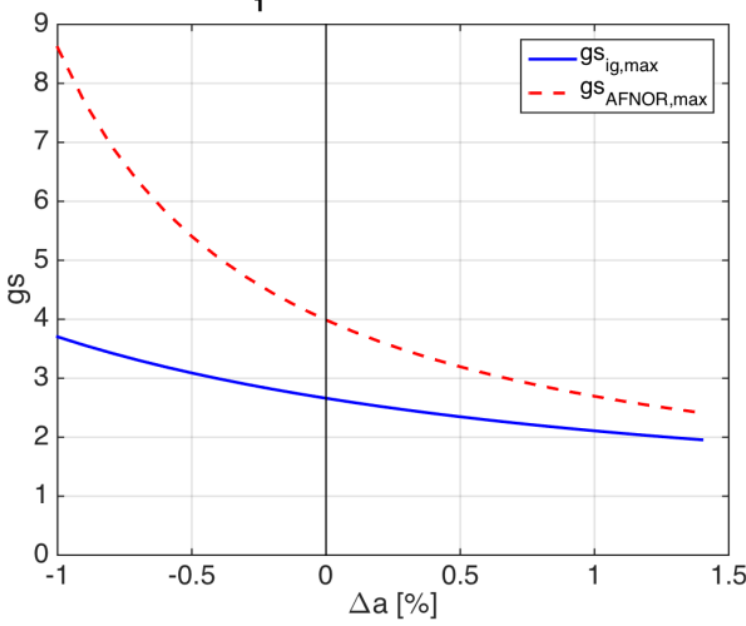

Figure 8: Specific sliding of the two wheels when using the criterion of balancing maximum values of $g s$ as well as the maximum specific sliding as a function of center distance variation, $\Delta a$, for a pinion with 17 teeth and a speed ratio of 2

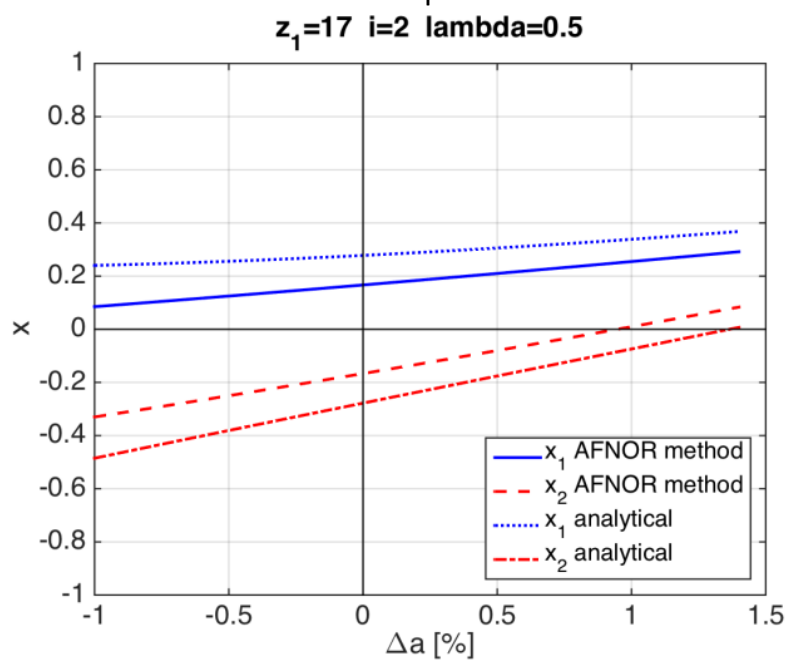

Figure 9: Profile shift values, $x_{1}$ and $x_{2}$, when using the criterion of balancing maximum values of specific sliding, as well as the maximum specific sliding, as a function of $\Delta a$, for a pinion with 17 teeth and a speed ratio of 2

This last case deliberately does not follow the recommendation for the value of $\lambda$. 
The analytical solution presented in this work and the approximate solution obtained by AFNOR give very close results when the value of $\lambda$ is correctly chosen.

It is also noticeable that, for the situation of null center distance variation $(\Delta a=0 \%), x_{1}=-x_{2}$, as can be seen from the system of equations (5). This is found both in the AFNOR and in the analytical balancing of the maximum values of $g_{s}$, as noticed in the several figures presented. It will also be seen that there is a point where the profile shift values of the pinion and of the wheel obtained through AFNOR procedure are equal to the analytical values calculated imposing the balancing of the maximum values of $g_{s}$.

After looking at the graphs of $x_{1}$ and $x_{2}$ for the various cases, it is clear that both variables follow a tendency that strongly resembles linear functions of the variable $\Delta a$. It is then possible to approximate $x_{1}$ and $x_{2}$ by a linear regression defined as

$$
\begin{aligned}
& x 1 \cong m_{1} \Delta a+b_{1} \\
& x 2 \cong m_{2} \Delta a+b_{2}
\end{aligned}
$$

where

$$
\begin{gathered}
m_{1}=\frac{n \sum_{i=1}^{n} x_{1 i} \Delta a_{i}-\sum_{i=1}^{n} x_{1 i} \times \sum_{i=1}^{n} \Delta a_{i}}{n \sum_{i=1}^{n} x_{1}^{2}-\left(\sum_{i=1}^{n} x_{1_{i}}\right)^{2}} \\
b_{1}=\frac{1}{n}\left(\sum_{i=1}^{n} \Delta a_{i}-m_{1} \sum_{i=1}^{n} x_{1_{i}}\right) \\
m_{2}=\frac{n \sum_{i=1}^{n} x_{2 i} \Delta a_{i}-\sum_{i=1}^{n} x_{2 i} \times \sum_{i=1}^{n} \Delta a_{i}}{n \sum_{i=1}^{n} x_{2}^{2}-\left(\sum_{i=1}^{n} x_{2 i}\right)^{2}} \\
b_{2}=\frac{1}{n}\left(\sum_{i=1}^{n} \Delta a_{i}-m_{2} \sum_{i=1}^{n} x_{2 i}\right)
\end{gathered}
$$

This approximation allows to store the information given by the analytical procedure with only four numbers, $m_{1}, b_{1}, m_{2}$ and $b_{2}$.

The graphs of the linear approximation of the profile shifts and the coefficient of determination, $R^{2}$, for the same gears used before to demonstrate the AFNOR procedure, as given by

$$
R^{2}=\frac{S_{t}-S_{r}}{S_{t}}
$$

where $S_{t}$ is the total sum of the squares of the residual between the data points and the mean

$$
S_{t}=\sum_{i=1}^{n}\left(x_{j, i}-\bar{x}_{j}\right)^{2}
$$

and $S_{r}$ is the sum of the squares of the residuals

$$
S_{r}=\sum_{i=1}^{n}\left(x_{j, i}-m_{j} \cdot \Delta a_{i}-b_{j}\right)^{2}
$$

where $j=1$ for the pinion, and $j=2$ for the wheel, and $i$ is the number result samples for each study case (Chapra and Canale 1989). 


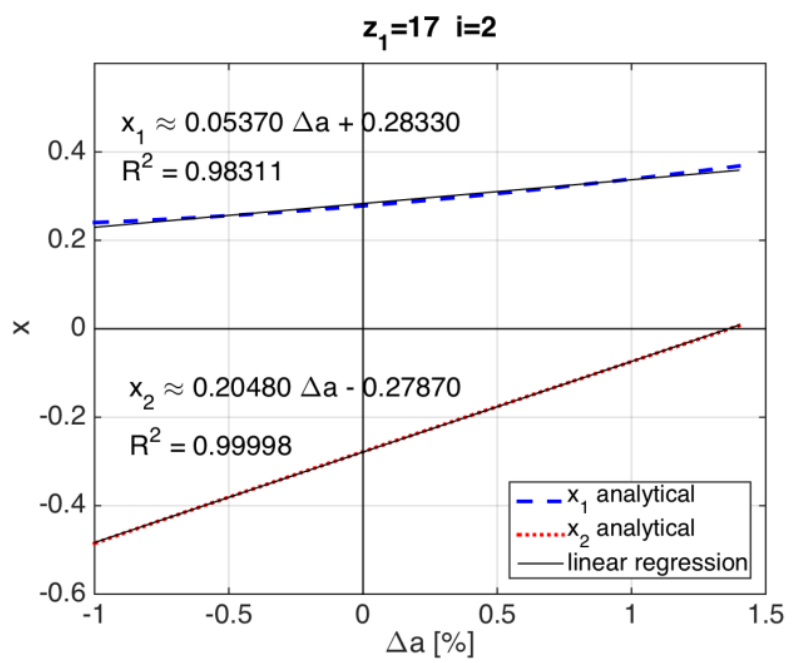

Figure 10: Profile shift values, $x_{1}$ and $x_{2}$, when using the criterion of balancing maximum values of specific sliding, as well as it's linear regression approximation, as a function of $\Delta a$, for a pinion with 17 teeth and a speed ratio of 2

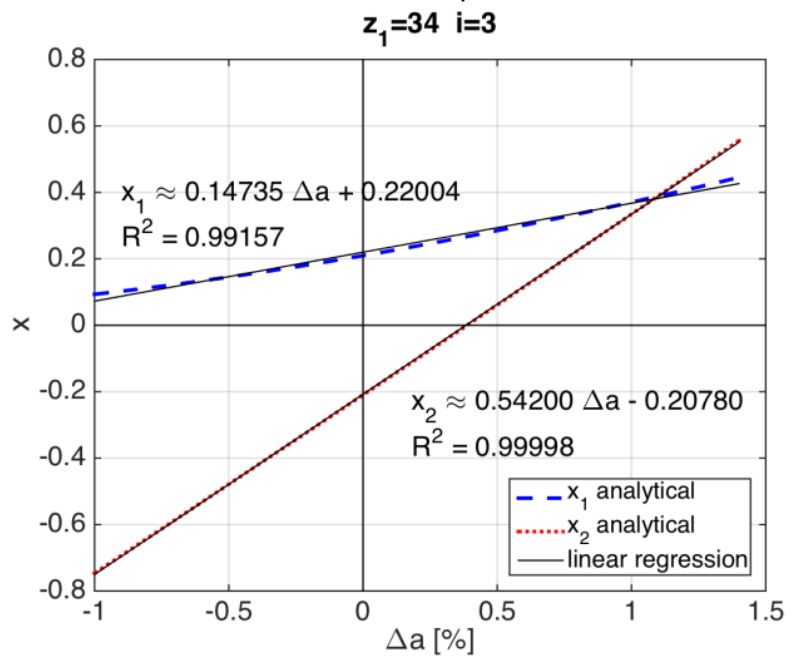

Figure 11: Profile Shift values, $x_{1}$ and $x_{2}$, when using the criterion of balancing maximum values of specific sliding, as well as its linear regression approximation, as a function of $\Delta a$, for a pinion with 34 teeth and a speed ratio of 3

Figures 10 and 11 correspond to the gears pairs with $z_{1}=17, i=2$ and $z_{1}=34, i=3$ respectively. Repeating this process to various gears, each one defined by $z_{1}$ and $i$, permits the construction of 4 tables, each one for $m_{1}, b_{1}, m_{2}$ and $b_{2}$. These four tables capture every solution, without ambiguity, for the output variables $x_{1}$ and $x_{2}$ for any combination of $z_{1}$ and $i$ working with a given $\Delta a$.

\begin{tabular}{|cc|ccc|}
\hline $\mathbf{m}_{\mathbf{1}}$ & & & $\mathbf{i}$ & \\
& & $\mathbf{2}$ & $\mathbf{3}$ & $\mathbf{4}$ \\
\hline & 17 & 0.05370 & 0.04850 & 0.04913 \\
$\mathbf{z}_{1}$ & 22 & 0.08347 & 0.07789 & 0.07643 \\
& 29 & 0.12387 & 0.11871 & 0.11731 \\
& 34 & 0.15200 & 0.14735 & 0.14617 \\
\hline
\end{tabular}

Table 1: Values of $m_{1}$ for different pinion teeth, $z_{1}$, and speed ratio, $i$ 


\begin{tabular}{|cc|ccc|}
\hline $\mathbf{b}_{1}$ & & & $\mathbf{i}$ & \\
& & $\mathbf{2}$ & $\mathbf{3}$ & $\mathbf{4}$ \\
\hline \multirow{4}{*}{$\mathbf{z}_{1}$} & 17 & 0.28330 & 0.36640 & 0.40083 \\
& 22 & 0.23390 & 0.30506 & 0.33921 \\
& 29 & 0.18857 & 0.24865 & 0.27853 \\
& 34 & 0.16608 & 0.22004 & 0.24741 \\
\hline
\end{tabular}

Table 2: Values of $b_{1}$ for different pinion teeth, $z_{1}$, and speed ratio, $i$

\begin{tabular}{|c|c|c|c|c|}
\hline \multirow[t]{2}{*}{$\mathrm{m}_{2}$} & & \multicolumn{3}{|c|}{$\mathbf{i}$} \\
\hline & & 2 & 3 & 4 \\
\hline \multirow{4}{*}{$z_{1}$} & 17 & 0.20480 & 0.29620 & 0.38332 \\
\hline & 22 & 0.25107 & 0.36816 & 0.48113 \\
\hline & 29 & 0.31711 & 0.46927 & 0.61766 \\
\hline & 34 & 0.36502 & 0.54200 & 0.71552 \\
\hline
\end{tabular}

Table 3: Values of $\mathrm{m}_{2}$ for different pinion teeth, $z_{1}$, and speed ratio, $i$

\begin{tabular}{|cc|ccc|}
\hline b2 & & & $\mathbf{i}$ & \\
& & $\mathbf{2}$ & $\mathbf{3}$ & $\mathbf{4}$ \\
\hline & 17 & -0.27870 & -0.35830 & -0.39420 \\
& 22 & -0.22796 & -0.29713 & -0.32931 \\
& 29 & -0.18073 & -0.23820 & -0.26547 \\
& 34 & -0.15690 & -0.20780 & -0.23210 \\
\hline
\end{tabular}

Table 4: Values of b2 for different pinion teeth, $z 1$, and speed ratio, $i$

It should be mentioned that in some cases, some results of $x_{1}$ and $x_{2}$ given by the analytical procedure are complex numbers. This happened in the cases of small $z_{1}, z_{1} \leq 15$, particularly for $\Delta a<0$. It was not given much importance to this phenomenon, since it only occurs in a small part of the possible range of $z_{1}$ values.

\section{Conclusions}

The question of imposed center distance requires appropriate choice of profile shift values $x_{1}$ (for the pinion) and $x_{2}$ (for the wheel). The approximate procedure documented by AFNOR (called by Henriot the 'ISO procedure') gives $x_{1}$ and $x_{2}$ leading to values of the maximum specific sliding of the wheel and of the pinion that are reasonably similar and close to the ideal value. The ideal, minimum value, may be obtained analytically imposing the balance of $g s_{i g, \text { max }}$ values.

However, the AFNOR procedure is not a good approximation for the whole field of center distance values, since, in some cases, a large difference is found in $g s$ when applying the two criteria (AFNOR and analytical balancing of $g s$ ).

When using the AFNOR procedure, the value of the parameter $\lambda$ is loosely chosen without much rigor.

The alternative propose presented in this paper's results and discussion do not present the lack of rigor across the field of center distance values and simultaneously eliminate the ambiguity of the parameter $\lambda$.

\section{References}

Association Française de Normalisation - AFNOR. 1980. AFNOR NF-E 23-013:1980 - Déport des Dentures de Roues Cylindriques pour Engrenages Réducteurs. La Plaine Saint-Denis: AFNOR.

Chapra, Steven C. and Raymond P. Canale. 1989. Numerical methods for engineers. $2^{\text {nd }}$ ed. New York: McGraw- Hill Book Company. 
Henriot, Georges. 2002a. "Engrenages Parallèles: Dentures corrigées". In Techniques de I'Ingénieur, BM 5621.9 - BM 5621.11. Saint-Denis Cedex: Techniques de l'Ingénieur.

- - - 2002b. "Engrenages Parallèles: Étude géométrique". In Techniques de I'Ingénieur, BM 5620.16 - BM 5620.17. Saint-Denis Cedex: Techniques de l'Ingénieur.

- - . 2002c. "Engrenages: définition, dessin et calcul". In Techniques de I'Ingénieur, B 636.16 - B 636.25. Saint-Denis Cedex: Techniques de l'Ingénieur.

- - . 2007. Engrenages conception, fabrication, mise en oeuvre. $8^{\text {th }}$ ed. Paris: Dunod. 